\title{
Comparison of long-term mortality risk following normal exercise vs adenosine myocardial perfusion SPECT
}

\author{
Alan Rozanski, MD, ${ }^{\text {a }}$ Heidi Gransar, MS, ${ }^{\mathrm{b}}$ Sean W. Hayes, MD, , c, \\ John D. Friedman, MD, MPH, , ${ }^{\mathrm{b}, \mathrm{c}}$ Rory Hachamovitch, $M D{ }^{\mathrm{d}}$ \\ and Daniel S. Berman, $M D^{\mathrm{b}, \mathrm{c}}$
}

Background. A higher frequency of clinical events has been observed in patients undergoing pharmacological vs exercise myocardial perfusion single-photon emission computed tomography (SPECT). While this difference is attributed to greater age and co-morbidities, it is not known whether these tests also differ in prognostic ability among patients with similar clinical profiles.

Methods and Results. We assessed all-cause mortality rates in 6,069 patients, followed for $10.2 \pm 1.7$ years after undergoing exercise or adenosine SPECT. We employed propensity analysis to match exercise and adenosine subgroups by age, gender, symptoms, and coronary risk factors. Within our propensity-matched cohorts, adenosine patients had an annualized mortality rate event rates that was more than twice that of exercise patients $(3.9 \%$ vs $1.6 \%$, $P<.0001)$. Differences in mortality persisted among age groups, including those $<55$ years old. In the exercise cohort, mortality was inversely related to exercise duration, with comparable mortality noted for patients exercising $<3 \mathrm{~min}$ and those undergoing adenosine testing.

Conclusions. Among patients with normal stress SPECT tests, those undergoing adenosine testing manifest a mortality rate that is substantially higher than that observed among adequately exercising patients, but comparable to that observed among very poorly exercising patients. This elevated risk underscores an important challenge for managing patients undergoing pharmacological stress testing. (J Nucl Cardiol 2010;17:999-1008.)

Key Words: Exercise $\cdot$ pharmacological stress $\cdot$ prognosis $\cdot$ coronary disease $\cdot$ myocardial perfusion SPECT

\section{See related editorial, pp. 983-984}

From the Division of Cardiology, ${ }^{\text {a }}$ St. Lukes Roosevelt Hospital, New York, NY; Departments of Imaging and Medicine, Cedars-Sinai Medical Center, ${ }^{\mathrm{b}}$ The Burns and Allen Research Institute, Los Angeles, CA; David Geffen School of Medicine, ${ }^{\mathrm{c}}$ University of California, Los Angeles, CA; and Department of Medicine, ${ }^{\mathrm{d}}$ University of Southern California, Los Angeles, CA.

Received for publication May 10, 2010; final revision accepted Sep 28, 2010.

Reprint requests: Daniel S. Berman, MD, Departments of Imaging and Medicine, Cedars-Sinai Medical Center, The Burns and Allen Research Institute, 8700 Beverly Building, Room 1258, Los Angeles, CA 90048; bermand@cshs.org.

$1071-3581 / \$ 34.00$

Copyright (c) 2010 The Author(s). This article is published with open access at Springerlink.com

doi:10.1007/s12350-010-9300-9

\section{INTRODUCTION}

Stress testing using single-photon emission computed tomography (SPECT) for myocardial perfusion imaging is currently widely used to guide many common medical management decisions, such as the need for cardiac catheterization among diagnostic patients or the need for myocardial revascularization among patients with known coronary artery disease (CAD). ${ }^{1} \mathrm{~A}$ basis of such use is the understanding that a normal stress SPECT study reliably identifies low-risk patients who can be successfully managed without the need for aggressive interventions. With respect to exercise SPECT, repeated investigation has confirmed that normal SPECT studies define patients with $<1 \%$ annualized event rate for cardiac events. ${ }^{1,2}$ It is widely assumed that normal pharmacological SPECT studies also identify low-risk patients, yet meta-analysis of this issue 
indicates that normal pharmacological stress studies are associated with somewhat higher event rates compared to those reported for exercise SPECT. ${ }^{3}$ This difference has been attributed to the greater age and co-existing morbidities that are usually present among patients who require pharmacological stress testing in lieu of exercise. However, is it possible that pharmacological stress simply limited in its ability to identify patients who are at low risk for cardiac events? Given the now large percent of SPECT studies currently performed with pharmacological stress, such a finding could be of great management significance. To examine this issue, we applied propensity analysis to compare long-term mortality among age and risk profile-matched patients manifesting a normal scintigraphic study during exercise vs adenosine myocardial perfusion SPECT.

\section{METHODS}

\section{Study Design}

We identified 8,504 consecutive patients undergoing physician-ordered stress/rest dual SPECT at Cedars Sinai Medical Center (CSMC) who were free of known CAD (no prior myocardial infarction, bypass surgery, or percutaneous coronary intervention) or of a prior clinical diagnosis of cardiomyopathy (i.e., known LV dysfunction due to ischemic or non-ischemic causes). We excluded 2,435 patients who manifested evidence of myocardial ischemia on SPECT imaging or who required testing using dobutamine stress. The remaining 6,069 patients with normal myocardial perfusion SPECT studies constituted our patient population. They had a mean age of $62 \pm 13$ years and 3,122 $(51 \%)$ male patients. All patients were prospectively enrolled in a research database at the time of testing and were followed for the occurrence of adverse clinical events. The study was approved by the CSMC Institutional Review Board.

\section{Historical Variables}

All patients completed a questionnaire to elicit information regarding personal demographics, chest pain symptoms, cardiac risk factors, and medication use. From the answer to three questions regarding chest pain (location, precipitants, and relief with rest or nitroglycerin), we identified four chest pain categories (asymptomatic, non-anginal chest pain, atypical, and typical angina). We also identified which patients complained of dyspnea only. ${ }^{4}$

\section{Imaging and Stress Protocol}

Exercise testing was performed using the Bruce protocol exercise. For exercising patients in whom difficulty with exercise was anticipated, we employed a modified Bruce protocol with exercise beginning at $1.7 \mathrm{mph}$ at zero incline (1 min) and then increased to a $5 \%$ incline $(1 \mathrm{~min})$ followed by the usual Bruce protocol (beginning at $1.7 \mathrm{mph}$ at a $10 \%$ incline). Heart rate, systolic and diastolic blood pressure measurements, and ECG tracings were obtained at rest and monitored during and after stress testing. The decision to perform exercise or adenosine testing was primarily based on the request of the referring physicians, but the final choice of protocol was modified as deemed appropriate by our supervising personnel. Patients who began exercise but who could not achieve $85 \%$ of their maximal predicted heart rate were generally converted into adenosine studies. The most common reasons for performing adenosine testing included patient inability to exercise due to co-morbid medical conditions (e.g., musculoskeletal problems or impaired gait) or history of poor exercise conditioning; failure to withhold beta-blocking medication; high resting blood pressure; and the presence of left bundle block or paced rhythm.

Patients were injected intravenously with thallium-201 (Tl-201) (3.0-4.5 mCi) at rest and Tc-99m sestamibi (25$40 \mathrm{mCi}$ ) at stress, using weight-adjusted doses. Rest Tl-201 SPECT was initiated $10 \mathrm{~min}$ after radionuclide injection and afterward, symptom-limited treadmill exercise was performed in 4,557 (75\%) patients and adenosine infusion testing was performed in 1,512 (25\%) patients.

Exercising patients were stopped before reaching exhaustion only for severe angina, sustained ventricular tachycardia, hemodynamically significant supraventricular dysrhythmias, or exertional hypotension. Radioisotope was injected at near maximal exercise. When tolerated, exercise was continued at maximal workload for an additional minute and then at a reduced workload for an additional $2 \mathrm{~min}$. Tc-99m sestamibi SPECT imaging was begun 15-30 min after exercise radiopharmaceutical injection. ${ }^{5}$

For adenosine testing, Tc-99m sestamibi was injected at the end of the third minute or at the end of the second minute of 5- or 6-min adenosine infusions, respectively $(140 \mathrm{mcg} / \mathrm{kg} /$ min for both). ${ }^{6}$ Our "adeno walk" protocol for concomitant low-level treadmill exercise was only performed in $146(10 \%)$ of our adenosine patients because this protocol was not instituted until 1995-1996. In the patients not undergoing the adeno-walk, SPECT imaging was delayed for approximately 60 min after injection during the adenosine infusion. Patients undergoing adenosine testing were instructed not to consume caffeine containing products for $24 \mathrm{~h}$ prior to testing, and they were routinely queried as to their adherence to this instruction prior to testing by our supervisory personnel. For patients ingesting caffeine containing products within $24 \mathrm{~h}$ of stress testing, the SPECT study was either re-scheduled or converted into a dobutamine stress test. Both exercise and adenosine patients were instructed to withhold beta-blocking medication for $48 \mathrm{~h}$ and calcium blockers for $24 \mathrm{~h}$ prior to testing, but among exercise patients, $8.3 \%$ were tested under the influence of calcium blockers and $6 \%$ under the influence of betablockers, and among the adenosine patients, $12 \%$ and $11 \%$ were tested under the influence of calcium and beta blockers, respectively ( $P<.001$ for both vs exercise, respectively).

\section{SPECT Acquisition Protocol}

SPECT studies were performed on multidetector scintillation cameras using an elliptical $180^{\circ}$ acquisition for $60-64$ 
projections at $20 \mathrm{~s}$ per projection. ${ }^{5}$ For Tl-201, two energy windows were used, including a $30 \%$ window centered on the 68 - to $80-\mathrm{keV}$ peak and a $10 \%$ window centered on the $167 \mathrm{keV}$ peak. For Tc-99m sestamibi SPECT, a 15\% window centered on the $140-\mathrm{keV}$ peak was used, and images were obtained in both supine and prone positions. ${ }^{7}$ Images were acquired using a $64 \times 64$ image matrix and were subject to quality control measures as previously described.

\section{SPECT Interpretation}

Semi-quantitative visual interpretation was performed using 20 segments for each image set. Segments were scored by consensus of two experienced observers. This interpretation was performed first blinded to the clinical information and then could be minimally modified based on the clinical data available as previously described, ${ }^{8}$ using a five-point score $(0$ = normal, 1 = equivocal, $2=$ moderate, $3=$ severe reduction of radioisotope uptake, and $4=$ absence of detectable tracer uptake in a segment). ${ }^{5}$ Summed stress scores (SSS) and summed rest scores were obtained by adding the scores of the 20 segments of the respective images. An SSS $\geq 4$ was used to define the presence of an abnormal study. Scintigraphic left ventricular size was subjectively categorized as either no, equivocal, or definite enlargement. The SPECT studies were also assessed visually for the presence of transient ischemic dilation (TID) of the left ventricle on the ungated SPECT images (scored as yes, no, equivocal, and only yes being considered present), with support of the visual interpretation coming from use of a previously described commercially available automated program (QPS, Cedars-Sinai, Lost Angeles, CA, USA). ${ }^{9}$

\section{Follow-up}

We ascertained overall all-cause mortality in our patient population, based on the analysis of the Social Security Death Index (SSDI). Average follow-up time was $10.2 \pm 1.7$ years. We also evaluated the results of available shorter-term followup (mean of $2.6 \pm 2.1$ years) to assess the frequency of hard cardiac events, which included cardiac death and non-fatal myocardial infarction, as evidenced by the appropriate combination of patient signs, symptoms, ECG changes, and enzyme elevations; during this time, 150 patients $(2.5 \%)$ were lost to cardiac follow-up. The confirmation of cardiac death was ascertained through examination of the records obtained through the blinded examination of our hospital-based patient information system (WebVS) and death certificates for all who died in Los Angeles County. Information regarding the performance and timing of revascularization procedures was also obtained, but was not used to censor patients with respect to allcause mortality or cardiac death since most studies employing the SSDI would not normally have access to such data.

\section{Statistical Analyses}

All data were analyzed using Stata software version 11 [StataCorp. 2005. Stata Statistical Software: Release 11.
College Station, TX: StataCorp LP]. Continuous variables were compared using the Wilcoxon rank-sum test because assumptions of the $t$-test were not met. Categorical variables were compared using Pearson $\chi^{2}$ test or Fisher's Exact test if cell counts of $<6$ were present. The $\chi^{2}$ test for trend was used when testing for trend. Survival curves were compared using the log-rank test or a test for trend across ordered groups, and Kaplan-Meier curves were graphed. Event rates were calculated by dividing the number of events by person-years, and compared across groups using the log-rank test. Twenty-four patients who underwent early revascularization procedures (bypass surgery or percutaneous coronary intervention) within the first 60 days after stress testing were censored for assessing the shorter-term cardiovascular outcomes. However, we did not censor patients undergoing early myocardial revascularization for the assessment of long-term mortality. Propensity matching was done using STATA's pscore ${ }^{10}$ and psmatch commands. Propensity scores were obtained ${ }^{10,11}$ to match nonischemic exercise to adenosine patients, based on age, gender, chest pain symptoms, and coronary disease risk factors. In order to create propensity-matched groups which had a low incidence of co-existing cardiac morbidities, we excluded patients with paced rhythm, left bundle branch block, atrial fibrillation, taking digoxin, manifesting TID, and those who had left ventricular enlargement or shortness of breath from this propensity analysis. The propensity scores were derived from the predicted probabilities of being in the adenosine vs the exercise group in a logistic regression model where the covariates were the above-mentioned matching variables (see Appendix). The balancing property of the propensity score was tested by stratifying into blocks and confirming that the mean propensity score was not significantly different in each block for adenosine and exercise patients. ${ }^{10}$ The matching was done using the single nearest-neighbor without caliper matching, with no replacement. ${ }^{11}$ In the propensity-matched patients, we compared continuous variables using the Wilcoxon signed-rank test, and we compared categorical variables using McNemar's test or McNemar's Exact test for small cell counts $<6$.

\section{RESULTS}

Table 1 compares the clinical characteristics of the exercise and adenosine SPECT groups. The adenosine patients were older, had fewer males, greater BMI, a greater mean pre-test likelihood of CAD, and they contained more patients with chest pain symptom. The adenosine patients also had a greater frequency of diabetes and hypertension, lower frequency of smokers, higher resting mean heart rate and systolic blood pressure, and more frequent LBBB, LVH, and atrial fibrillation, and less frequent left ventricular enlargement. During a mean follow-up of $10.2 \pm 1.7$ years (range $7.5-14$ years), 978 patients $(16.1 \%)$ died, including $466(10 \%)$ of the exercise patients, and 512 (34\%) of the adenosine patients. The annualized mortality event rate was markedly higher in the adenosine patients compared to the exercising patients $(4.3 \%$ [95\% CI: 
Table 1. Comparison of exercise and adenosine patients

\begin{tabular}{|c|c|c|c|}
\hline & Exercise $(N=4,557)$ & Adenosine $(\mathrm{N}=1,512)$ & P-values* \\
\hline Age & $59.9 \pm 12.4$ & $68.8 \pm 12.1$ & $<.0001$ \\
\hline Male & $2585(56.7 \%)$ & $537(35.5 \%)$ & $<.001$ \\
\hline Chest pain symptoms & & & $<.001$ overall \\
\hline Asymptomatic & $1478(32.4 \%)$ & $433(28.6 \%)$ & .006 \\
\hline Non-anginal chest pain & $1270(27.9 \%)$ & $342(22.6 \%)$ & $<.001$ \\
\hline Atypical angina & $1245(27.3 \%)$ & $466(30.8 \%)$ & .009 \\
\hline Typical angina & $423(9.3 \%)$ & $167(11.0 \%)$ & .045 \\
\hline Dyspnea only & $140(3.1 \%)$ & $104(6.9 \%)$ & $<.001$ \\
\hline \multicolumn{4}{|l|}{ CAD risk factors } \\
\hline Diabetes & $344(7.6 \%)$ & $233(15.4 \%)$ & $<.001$ \\
\hline Hypercholesterolemia & 1977 (43.4\%) & $560(37.0 \%)$ & $<.001$ \\
\hline Smoking & $674(14.8 \%)$ & $191(12.6 \%)$ & .04 \\
\hline Family history & $1148(25.2 \%)$ & $298(19.7 \%)$ & $<.001$ \\
\hline Hypertension & $1786(39.2 \%)$ & $843(55.8 \%)$ & $<.001$ \\
\hline Body mass index & $26.3 \pm 4.5$ & $26.7 \pm 5.5$ & .03 \\
\hline Claudication & $7(0.2 \%)$ & $4(0.3 \%)$ & .48 \\
\hline Pre-test CAD likelihood & $33.9 \pm 29.1$ & $44.3 \pm 30.0$ & $<.0001$ \\
\hline \multicolumn{4}{|l|}{ Medications while testing } \\
\hline Beta-blockers & $263(5.8 \%)$ & $165(10.9 \%)$ & $<.001$ \\
\hline Calcium-blockers & $376(8.3 \%)$ & $188(12.4 \%)$ & $<.001$ \\
\hline Digoxin & $191(4.2 \%)$ & $115(7.6 \%)$ & $<.001$ \\
\hline \multicolumn{4}{|l|}{ Hemodynamics and ECG findings } \\
\hline Resting heart rate & $68.8 \pm 12.5$ & $72.1 \pm 14.2$ & $<.0001$ \\
\hline Resting systolic BP & $137.6 \pm 21.5$ & $149.8 \pm 27.7$ & $<.0001$ \\
\hline Resting diastolic BP & $84.9 \pm 10.6$ & $80.2 \pm 12.6$ & $<.0001$ \\
\hline Left bundle branch block & $39(0.9 \%)$ & $63(4.2 \%)$ & $<.001$ \\
\hline Paced rhythm & $30(0.7 \%)$ & $55(3.6 \%)$ & $<.001$ \\
\hline Left ventricular hypertrophy & $390(8.6 \%)$ & $214(14.2 \%)$ & $<.001$ \\
\hline Left ventricular enlargement & $266(5.9 \%)$ & $45(3.0 \%)$ & $<.001$ \\
\hline Arial fibrillation & $53(1.2 \%)$ & $57(3.8 \%)$ & $<.001$ \\
\hline
\end{tabular}

*All $P$-values for continuous variables were Wilcoxon rank-sum test, and Pearson $\chi^{2}$ test for categorical variables. $C A D$, Coronary artery disease; $B P$ blood pressure.

$4.0 \%-4.7 \%$ ] vs $1.1 \%$ [95\% CI: $1.0 \%-1.1 \%$ ], $P<.0001$ ). This difference in mortality rates was present both for adenosine vs exercise patients tested without influence of beta-blocking medications $(4.5 \%$ vs $1.0 \%, P<.0001)$ and those tested under the influence of beta-blocking medication $(3.1 \%$ vs $1.2 \%, P<.0001)$. Likewise the annual mortality rate was $4.2 \%$ vs $1.0 \%(P<.0001)$ and $5.3 \%$ vs $1.5 \%(P<.0001)$ for adenosine vs exercise patients, for those not under the influence and those under the influence of calcium channel blockers, respectively.

The presence of TID was noted among $7(0.2 \%)$ of the exercise patients and $7(0.5 \%)$ of the adenosine patients $(P=.03)$, and in both groups, the annualized mortality rate (presented with confidence intervals) was higher for those with TID than those without: $4.8 \%$ $(1.5 \%-14.9 \%)$ vs $1.0 \%(1.0 \%-1.1 \%), P=.003$, for the exercise patients, and $7.9 \%(3.0 \%-21.2 \%)$ vs $4.3 \%$ (3.9\%-4.7\%), $P=.23$, for the adenosine patients.

Among the exercise patients, $839(18 \%)$ had ST depression during testing, whereas only 61 (4\%) had ST depression during adenosine infusion. The annualized mortality in the exercise and adenosine patients with ST segment depression was $(0.8 \%$ and $2.6 \% /$ year, respectively, $P<.0001)$. In the exercise patients, $424(9 \%)$ had chest pain with exercise, compared to only 25 (2\%) adenosine patients, with annualized mortality rates of 1.1 and $1.9 \% / y e a r$ in these two groups, respectively, $P=.26$.

\section{Propensity-Based Comparisons}

We next used propensity analysis to match exercise and adenosine patients who did not have TID, LBBB, paced, afib, or were taking digoxin, or had left 
Table 2. Comparison of exercise and adenosine patients following propensity matching

\begin{tabular}{|c|c|c|c|}
\hline & Exercise $(\mathbf{N}=1,125)$ & Adenosine $(\mathrm{N}=1,125)$ & P-values * * \\
\hline Age $^{*}$ & $66.8 \pm 11.0$ & $67.4 \pm 12.0$ & .08 \\
\hline Male* & $411(36.5 \%)$ & $396(35.2 \%)$ & .49 \\
\hline \multicolumn{4}{|l|}{ Chest pain symptoms } \\
\hline Asymptomatic* & $320(28.4 \%)$ & $337(30.0 \%)$ & .57 \\
\hline Non-anginal chest pain* & $289(25.7 \%)$ & $259(23.0 \%)$ & .15 \\
\hline Atypical angina & $387(34.4 \%)$ & $389(34.6 \%)$ & .93 \\
\hline Typical angina & $129(11.5 \%)$ & $140(12.4 \%)$ & .46 \\
\hline \multicolumn{4}{|l|}{ CAD risk factors } \\
\hline Diabetes* & $140(12.4 \%)$ & $167(14.8 \%)$ & .10 \\
\hline Hypercholesterolemia* & $462(41.1 \%)$ & $435(38.7 \%)$ & .24 \\
\hline Smoking* & $150(13.3 \%)$ & $150(13.3 \%)$ & 1.00 \\
\hline Family history* & $227(20.2 \%)$ & $231(20.5 \%)$ & .83 \\
\hline Hypertension* & $618(54.9 \%)$ & $619(55.0 \%)$ & 1.00 \\
\hline Body mass index* & $26.5 \pm 5.0$ & $26.9 \pm 5.5$ & .09 \\
\hline Claudication & $1(0.1 \%)$ & $2(0.2 \%)$ & 1.00 \\
\hline Pre-test CAD likelihood & $40.4 \pm 28.8$ & $41.2 \pm 28.3$ & .55 \\
\hline \multicolumn{4}{|l|}{ Medications while testing } \\
\hline Beta-blockers* & $118(10.5 \%)$ & $116(10.3 \%)$ & .89 \\
\hline Calcium-blockers* & $129(11.5 \%)$ & $129(11.5 \%)$ & 1.00 \\
\hline \multicolumn{4}{|l|}{ Hemodynamics and ECG findings } \\
\hline Resting heart rate* & $71.9 \pm 13.9$ & $71.9 \pm 13.9$ & .92 \\
\hline Resting systolic BP* & $146.7 \pm 23.1$ & $148.7 \pm 27.2$ & .08 \\
\hline Resting diastolic BP* & $81.4 \pm 10.4$ & $80.1 \pm 12.5$ & .003 \\
\hline Left ventricular hypertrophy* & $144(12.8 \%)$ & $159(14.1 \%)$ & .35 \\
\hline
\end{tabular}

* Patients were matched on these characteristics (asymptomatic and non-anginal chest pain patients were grouped together for matching purposes).

* * All $P$-values for continuous variables were Wilcoxon matched-pairs signed-rank test, McNemar, or Exact McNemar test.

ventricular enlargement or shortness of breath. Matching was done on the basis of age (grouped for the purpose of matching by half-decade), gender, asymptomatic or nonanginal chest pain symptom, diabetes, hypercholesterolemia, smoking, family history of CAD, hypertension, body mass index, influence of beta-blockers or calciumblockers, resting heart rate, resting systolic and diastolic blood pressure, and left ventricular hypertrophy. The propensity model is presented in the Appendix. The propensity scores were matched to an average difference of 0.05 (median matching precision $=0.03$ ) and the largest difference was $<0.15$ between individual adenosine and matched exercise patients. The resultant clinical characteristics of the two propensity-matched subgroups are listed in Table 2. A small but statistically significant difference persisted in the average diastolic blood pressure between the two groups, but the groups were otherwise comparably matched in terms of clinical presentation, risk factor profiles, and Bayesian likelihood of CAD. Yet within these propensity-matched subgroups, the annualized mortality event rate remained substantially higher within the adenosine cohort (3.9\% [95\% CI: $3.5 \%-4.3 \%$ ] vs $1.6 \%$ [95\% CI: $1.4 \%-1.9 \%$ ], $P<.0001)$. A comparison of adjusted Kaplan-Meier estimates for mortality in the exercise and adenosine patients before and after propensity matching indicated an approximately fourfold higher rate of mortality prior to propensity-matching (Figure 1A), and twofold higher rate following propensity matching (Figure $1 \mathrm{~B}$ ).

To assess if these differences varied in younger vs older patients, we divided our matched patients into those who were $\leq 55,55-65$, and $>65$ years. Analysis of the Kaplan-Meir curves revealed that the difference in mortality rates among the normal SPECT exercise vs adenosine patients persisted in each age group (Figure 2).

Among the propensity-matched patients, comparison was also made between the adenosine and exercise groups after dividing the latter group according to exercise duration (Figure 3). As exercise duration diminished, mortality rate increased progressively. The annualized mortality rates for those exercising $\geq 9$, 

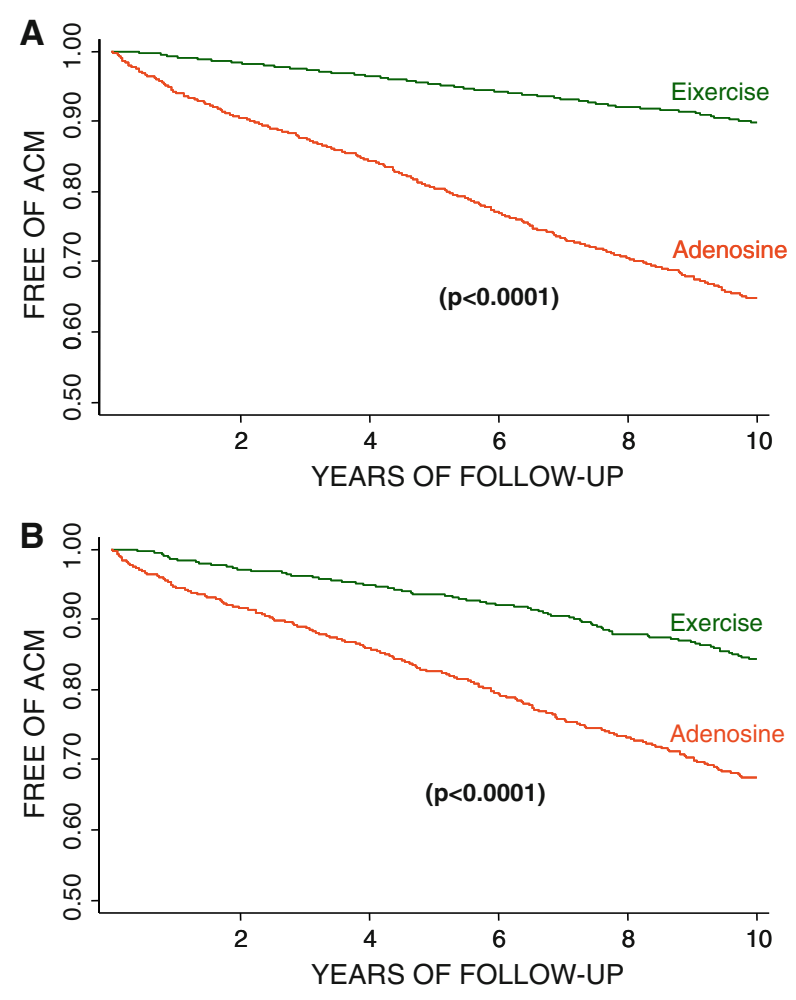

Figure 1. Comparison of probability of survival from death (y-axis) during 10-year follow-up from all causes in patients undergoing exercise vs adenosine SPECT. (A) Comparison of all exercise and adenosine patients in our study prior to propensity matching. (B) The comparative survival in exercise and adenosine patients following propensity-matching based on age, gender, chest pain symptom, and CAD risk factors. $A C M$, All-cause mortality.

$6-8.9,3-5.9$, and $<3$ min were $0.8 \%, 1.3 \%, 2.0 \%$, and $3.4 \%$, respectively (trend $P<.0001$ ). The mortality rate for patients unable to exercise for more than 3 min was similar to that observed among patients undergoing adenosine SPECT $(P=.65)$.

We also assessed outcomes among the 349 (8\%) of our exercising patients who underwent a modified Bruce protocol compared to the 4,208 (92\%) patients who underwent standard Bruce protocol exercise. The subgroup undergoing modified Bruce protocol exercise were older $(66.0 \pm 12.1$ vs $59.4 \pm 12.3$ years, $P<$ $.0001)$, had a lower mean exercise duration $(7.0 \pm 3.2$ vs $7.5 \pm 2.7 \mathrm{~min}, P=.003$ ), and a substantially higher annualized mortality event rate: $2.4 \%(1.9 \%-3.0 \%)$ vs $0.9 \%(0.8 \%-1.0 \%), P<.0001$. Comparable differences in event rates persisted for the exercise versus adenosine patient subgroups that did and did not have resting ECG abnormalities at rest $(P<.003$ for both pairwise comparisons).

We further assessed the comparative event rate in the propensity-matched groups, after excluding the patients with the following co-morbidities in addition to the ones that were excluded before:current smoking, diabetes, $\mathrm{BMI} \geq 30$, presence of $\mathrm{LVH}$, or peripheral vascular disease. Among the 606 exercise patients and 535 adenosine patients without any of these co-morbidities, the annual mortality rate remained significantly greater among the adenosine patients: $3.6 \%$ vs $1.5 \%$ $(P<.0001)$.

\section{Comparison of Short-Term Cardiac Outcomes}

Short-term cardiac events occurred among 44 of our adenosine patients, including 31 (with one censored) cardiac deaths and 13 non-fatal myocardial infarctions, as well as among 27 of our exercise patients, including 12 cardiac deaths and 15 non-fatal myocardial infarctions. The annualized cardiac event rate in the adenosine vs exercise groups was $1.2 \%$ vs $0.2 \%(P<.0001)$. Within the propensity-matched cohorts, the annualized cardiac event rate in the adenosine vs exercise patients also remained twofold higher: $1.1 \%$ vs $0.2 \%, P<.0001$.

\section{DISCUSSION}

In this study, we compared the long-term mortality rate among patients who had a normal stress-rest SPECT study during exercise study testing vs during adenosine infusion. Over our mean 10-year follow-up, adenosine patients had a markedly greater mortality rate than did exercise patients, but they were older, with more diabetes, hypertension, atrial fibrillation, and other clinical factors. Thus, we used propensity analysis to define a matched cohort of exercise and adenosine patients who had very similar clinical profiles. Our propensity-matched adenosine SPECT patients still had twice the annual mortality of their exercise counterparts.

Diminished sensitivity for ischemic detection by adenosine SPECT is an unlikely explanation for our findings. Early studies found that comparable magnitudes of myocardial ischemia were induced when experimental patients underwent both dipyridamole (the first vasodilator used in clinical practice) and exercise myocardial perfusion imaging. ${ }^{12,13}$ Subsequently, patients undergoing both adenosine and exercise SPECT were also found to have similar sensitivity and specificity for CAD detection. ${ }^{14,15}$

Prior follow-up studies have generally depended on relatively short-term follow ( $\sim 2-3$ years) to examine the "warranty period" associated with normal stress SPECT studies. Previously, we noted an annualized cardiac event rate that was elevated in our adenosine vs exercise patients, ${ }^{16}$ which we and others ${ }^{17,18}$ attributed to greater age and co-morbidity among adenosine 

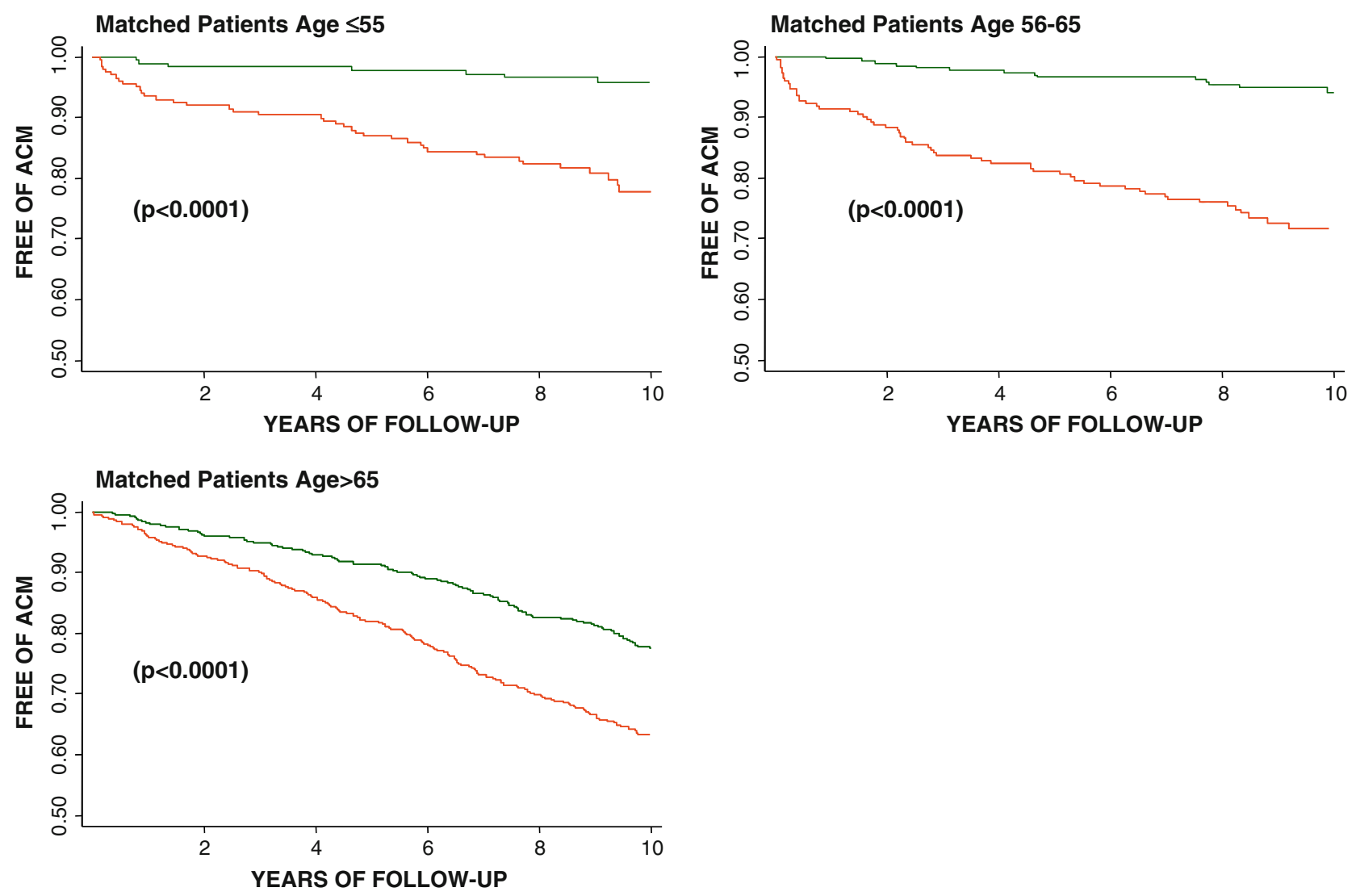

Figure 2. Comparative survival of the propensity-matched exercise and adenosine patients following division of these patients by age.

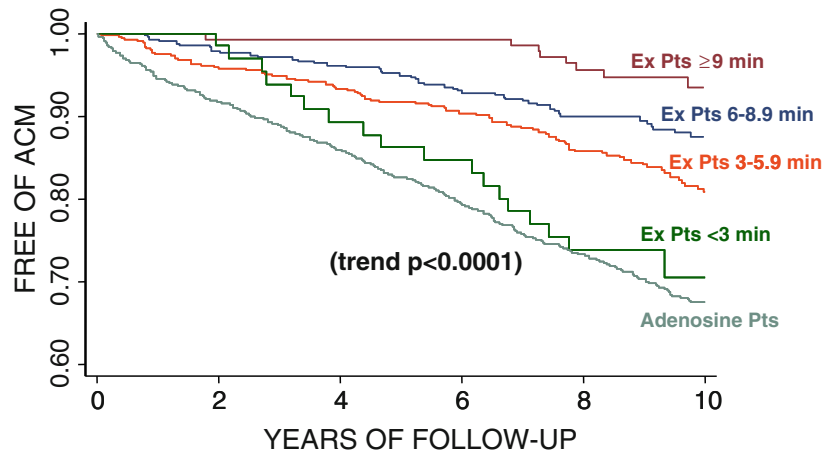

Figure 3. Comparison of survival in the propensity-matched exercise and adenosine patients, with the exercise patients divided according to exercise duration. Mortality rate increased progressively as exercise duration diminished. There was no statistical difference in mortality rate among patients unable to exercise $>3 \mathrm{~min}$ and patients undergoing adenosine SPECT $(P=.65)$.

patients. In a meta-analysis of 10 exercise and 8 pharmacological myocardial perfusion studies, Navare et $\mathrm{al}^{3}$ reported an annualized cardiac event of $1.78 \%$ for normal pharmacological studies vs only $0.65 \%$ for normal exercise studies. These investigators also suggested that the difference in this risk was attributable to differences in the a priori risk for cardiac risk in the exercise and pharmacological populations used for their meta-analysis. More recently, the follow-up of a large cohort of 16,854 consecutive patients with a normal stress SPECT study reported differences in annualized mortality which was comparable to that observed in our study: an annualized mortality rate of $0.6 \%$ for their exercise patients vs $4.1 \%$ for their patients undergoing 
pharmacological stress. ${ }^{19}$ Similarly, significant differences in event rates have also been noted for dobutamine vs exercise SPECT. ${ }^{20,21}$ However, none of these prior studies used propensity-analysis or other matching techniques which would have allowed them to compare patients with similar coronary risk profiles during pharmacological and exercise stress testing.

\section{Physiological Considerations}

Various conditions that inhibit exercise are likely to be preferentially concentrated among patients undergoing pharmacological stress testing. These include respiratory diseases; musculoskeletal impairment due to chronic inflammatory autoimmune diseases (e.g., rheumatoid arthritis) or degenerative musculoskeletal disorders (e.g., osteoarthritis); impaired gait, balance or walking ability stemming from cerebrovascular disease, degenerative disorders of the central nervous system, or peripheral vascular disease; fatigue or weakness due to a range of systemic illnesses or increasing frailty; and poor exercise conditioning, which may increase with age or obesity. Since we did not catalog such non-cardiac conditions at the time our patients underwent stress SPECT imaging, our propensity analyses could not exclude these potential co-morbidities as the cause for our findings. Nevertheless, various findings suggest more that unexamined co-morbidity as an explanation for our findings, including the strength of our propensity analyses and our finding of substantially higher mortality among propensity-matched patients of all age groups, including patients $<55$ years in age. Moreover, we also observed a marked difference in the rate of cardiac mortality among patients undergoing pharmacological vs exercise SPECT, even after excluding cardiac co-morbidities and risk factors, including patients with dyspnea, peripheral vascular disease, atrial fibrillation, LV enlargement, LVH, diabetes, or the presence of a high BMI.

Of note, the mortality rate in our pharmacological patients paralleled that of patients manifesting very poor exercise tolerance. Mortality in our exercise patients varied inversely with exercise duration. In part, the increased events among our patients with low exercise fitness may have been due to decreased sensitivity for ischemic detection by SPECT among patients failing to exercise adequately. ${ }^{2,23}$ However, poor exercise fitness and sedentary behavior also constitute increased risk for mortality due to their association with inflammation and oxidative stress, ${ }^{24,25}$ endothelial dysfunction ${ }^{25}$; metabolic syndrome and disturbed glucose metabolism, ${ }^{26}$ overweight $^{27}$; and adverse association with other cardiac risk factors, such as lower HDL levels ${ }^{28}$ and higher blood pressure. ${ }^{29}$ Similarly, these same pathophysiological factors would be expected to contribute to the increased mortality among those pharmacological patients in our study who did not perform exercise SPECT due to poor exercise tolerance.

\section{Limitations}

Our study has a number of limitations. Our study is a retrospective single-center study and patient matching was accomplished using statistical techniques but not prospectively selected similar groups. Moreover, as aforementioned, concomitant non-cardiac diseases which we did not assess may have contributed to differences in prognosis in our study. Similarly, prior reported studies concerning the prognostic efficacy of pharmacological SPECT have not stratified patients according to co-morbidities or the reasons for performing pharmacological rather than exercise myocardial perfusion SPECT. The other clinical information that is taken from our exercise studies may have slightly modified the interpretation of images while this information was not available with the interpretation of the adenosine studies. Finally, a factor that was unanticipated at the time of testing is the potential effect of betablockers and calcium channel blockers on reducing the presence and size of myocardial perfusion abnormalities on SPECT images. Several reports suggest that this effect may be substantial. ${ }^{30,31}$ However, the frequency of adenosine patients who were on calcium or betablockers at the time of testing was relatively small in our population, and the higher mortality rates among adenosine vs exercise patients persisted when such patients were eliminated from our analyses.

\section{Clinical Significance}

For years, normal stress myocardial perfusion imaging has been promulgated as providing a "warranty" for low cardiac risk. Subsequently, various subgroups have been identified as having increased risk despite non-ischemic SPECT responses, including patients with diabetes, ${ }^{32}$ atrial fibrillation, ${ }^{33}$ and dyspnea. ${ }^{4}$ However, even though some studies have also reported relatively increased events in patients undergoing pharmacological stress, ${ }^{3,16,17,19}$ by and large, patients with normal pharmacological stress tests are considered as having low cardiac risk, and in our Institution, these patients have manifested the same low referral rate to cardiac catheterization as do patients with normal exercise SPECT. Our findings of a significantly increased mortality risk in propensity-matched adenosine patients, even among younger propensity-matched subjects, raise an important potential caution to the easy assignment of normal pharmacological stress patients to 
a "low-risk" categorization. Rather, our findings suggest that patients with normal pharmacological stress tests are deserving of closer scrutiny over time compared to patients with normal exercise SPECT studies.

Our findings also indicate the need for further prospective study to identify the underlying reasons for the increased mortality among non-ischemic patients studied by pharmacological means. Such study would assess the role of various inflammatory diseases, occult subclinical atherosclerosis, poor physical fitness and sedentary lifestyle, and other factors in mediating the differences in outcome among exercise and adenosine patients. Future outcome studies should collect prospective information as to the reason(s) for performing pharmacological stress at the time of testing so that component factors that contribute to higher mortality among such patients can be better identified and studied.

\section{Acknowledgments}

This study was supported in part by grants from BristolMyers Squibb Medical Imaging, Inc., Billerica, MA, and Astellas, Inc., Deerfield, IL.

Dr Berman has grants from Lantheus Medical Imaging, Inc., is on the speaker bureau for Astellas Pharma, Inc., and participates in royalties to Cedars-Sinai Medical Center for licensure of software used in this study. Dr Hachamovitch has grants from Astellas Pharma, Inc., GE Healthcare, Siemens Medical Solutions, and Bracco, and is a consultant for Lantheus Medical Imaging.

The other authors have indicated that they have no financial conflicts of interest.

\section{Open Access}

This article is distributed under the terms of the Creative Commons Attribution Noncommercial License which permits any noncommercial use, distribution, and reproduction in any medium, provided the original author(s) and source are credited.

\section{APPENDIX}

Logistic regression model used for deriving propensity score, predicting having the adenosine test, vs having the exercise test

\begin{tabular}{lccc}
\hline \multicolumn{1}{c}{ Predictors } & OR & $\begin{array}{c}\mathbf{9 5 \%} \\
\text { Confidence } \\
\text { interval }\end{array}$ & P-value \\
\hline $\begin{array}{c}\text { Age group per half } \\
\text { decades }\end{array}$ & 1.20 & $(1.16-1.25)$ & $<.001$ \\
\begin{tabular}{l} 
Male gender \\
\hline
\end{tabular} & 0.73 & $(0.62-0.86)$ & $<.001$ \\
\hline
\end{tabular}

Appendix continued

\begin{tabular}{|c|c|c|c|}
\hline Predictors & OR & $\begin{array}{l}95 \% \\
\text { Confidence } \\
\text { interval }\end{array}$ & $P$-value \\
\hline BMI $\left(\mathrm{kg} / \mathrm{m}^{2}\right)$ & 1.05 & $(1.03-1.06)$ & $<.001$ \\
\hline $\begin{array}{l}\text { Asymptomatic or } \\
\text { non-anginal chest } \\
\text { pain }\end{array}$ & 0.81 & $(0.70-0.95)$ & .008 \\
\hline Diabetes & 1.53 & $(1.20-1.94)$ & $<.001$ \\
\hline High cholesterol & 0.75 & $(0.64-0.88)$ & $<.001$ \\
\hline Smoking & 1.31 & $(1.05-1.63)$ & .02 \\
\hline $\begin{array}{l}\text { Family history of } \\
\text { CAD }\end{array}$ & 0.90 & $(0.75-1.09)$ & .29 \\
\hline Beta-blockers & 1.64 & $(1.25-2.16)$ & $<.001$ \\
\hline Calcium-blockers & 0.87 & $(0.68-1.12)$ & .28 \\
\hline Hypertension & 1.46 & $(1.23-1.73)$ & $<.001$ \\
\hline $\begin{array}{l}\text { Resting heart rate } \\
\text { (beats } / \mathrm{min} \text { ) }\end{array}$ & 1.03 & $(1.02-1.03)$ & $<.001$ \\
\hline $\begin{array}{l}\text { Systolic blood } \\
\text { pressure }\end{array}$ & 1.03 & $(1.02-1.03)$ & $<.001$ \\
\hline $\begin{array}{l}\text { Diastolic blood } \\
\text { pressure }\end{array}$ & 0.92 & $(0.92-0.93)$ & $<.001$ \\
\hline LVH & 1.48 & $(1.16-1.89)$ & .001 \\
\hline
\end{tabular}

\section{References}

1. Klocke FJ, Baird MG, Lorell BH, Bateman TM, Messer JV, Berman DS, et al. ACC/AHA/ASNC guidelines for the clinical use of cardiac radionuclide imaging-executive summary: A report of the American College of Cardiology/American Heart Association Task Force on Practice Guidelines (ACC/AHA/ASNC Committee to Revise the 1995 Guidelines for the Clinical Use of Cardiac Radionuclide Imaging). J Am Coll Cardiol 2003;42:1318-33.

2. Metz LD, Beattie M, Hom R, Redberg RF, Grady D, Fleishchmann KE. The prognostic value of normal exercise myocardial perfusion imaging and exercise echocardiography. J Am Coll Cardiol 2007;49:227-37.

3. Navare SM, Mather JF, Shaw LJ, Fowler MS, Heller GV. Comparison if risk stratification with pharmacologic and exercise stress myocardial perfusion imaging: A meta-analysis. J Nucl Cardiol 2004;11:551-6.

4. Abidov A, Rozanski A, Hachamovitch R, Hayes SW, Aboul-Enein F, Cohen I, et al. Prognostic significance of dyspnea in patients referred for cardiac stress testing. N Engl J Med 2005;353:188998.

5. Berman DS, Kiat H, Friedman JD, Wang FP, Van Train K, Matzer L, et al. Separate acquisition rest thallium-201/stress technetium99m sestamibi dual-isotope myocardial perfusion single-photon emission computed tomography: A clinical validation study. J Am Coll Cardiol 1993;22:1455-64.

6. Amanullah AM, Kiat H, Friedman JD, Berman DS. Adenosine technetium-99m sestamibi myocardial perfusion SPECT-MPI in women: Diagnostic efficacy in detection of coronary artery disease. J Am Coll Cardiol 1996;27:803-9. 
7. Hayes SW, De Lorenzo A, Hachamovitch R, Dhar SC, Hsu P, Cohen I, et al. Prognostic implications of combined prone and supine acquisitions in patients with equivocal or abnormal supine myocardial perfusion SPECT. J Nucl Med 2003;44:1633-40.

8. Germano G, Berman DS, editors. Clinical gated cardiac SPECT. 2nd ed. Malden, MA: Blackwell Publishing; 2006.

9. Aiden A, Bax JJ, Hayes SW, Hachamovitch R, Cohen I, Gerlach J, et al. Transient ischemic dilation ratio of the left ventricle is a significant predictor of future cardiac events in patients with otherwise normal myocardial perfusion SPECT-MPI. J Am Coll Cardiol 2003;42:1818-25.

10. Becker SO, Ichino A. Estimation of average treatment effects based on propensity scores. Stata J 2002;2:358-77.

11. Leuven E, Sianesi B. PSMATCH2: Stata module to perform full Mahalanobis and propensity score matching, common support graphing, and covariate imbalance testing, 2003. http://ideas.repec. org/c/boc/bocode/s432001.html.

12. Leppo JA. Dipyridamole-Thallium imaging: The lazy man's stress test. J Nucl Med 1989;30:281-7.

13. Varma SK, Watson DD, Beller GA. Quantitative comparison of thallium-201 scintigraphy after exercise and dipyridamole in coronary artery disease. Am J Cardiol 1989;64:871-7.

14. Levine MG, Ahlberg AW, Mann A, White MP, McGill CC, de Leon CM, et al. Comparison of exercise, dipyridamole, adenosine, and dobutamine stress with the use of Tc-99m tetrofosmin tomographic imaging. J Nucl Cardiol 1999;6:389-96.

15. Gupta NC, Esterbrooks DJ, Hilleman DE, Mohiuddin SM. Comparison of adenosine and exercise thallium-201 single photon computed tomography (SCPECT) myocardial perfusion imaging. The GE SPECT Multicenter Adenosine Study Group. J Am Coll Cardiol 1992;19:248-57.

16. Hachamovitch R, Hayes S, Friedman JD, Cohen I, Shaw LJ, Germano G, Berman DS. Determinants of risk and its temporal variation in patients with normal stress myocardial perfusion scans: What is the warranty period of a normal scan? J Am Coll Cardiol 2003;41:1329-40.

17. Shaw LJ, Iskandrian AE. Prognostic value of gated myocardial perfusion SPECT-MPI. J Nucl Cardiol 2004;11:171-85.

18. Stratmann HG, Tamesis BR, Younis LT, Wittry MD, Miller DD. Prognosis value of dipyridamole technetium $99 \mathrm{~m}$ sestamibi myocardial tomography in patients with stable chest pain who are unable to exercise. Am J of Cardiol 1994;73:647-52.

19. Chang SU, Nabi F, Xu J, Raza U, Mahmarian JJ. Normal stressonly versus standard stress/rest myocardial perfusion imaging. J Am Coll Cardiol 2010;55:221-30.

20. Calnon DA, McGrath PD, Doss AL, Harrell FE, Watson DD, Beller GA. Prognostic value of dobutamine stress technetium99m-sestamibi single-photon emission computed tomography myocardial perfusion imaging:stratification of a high-risk population. J Am Coll Cardiol 2001;38:1151-517.

21. Navare SM, Katten D, Johnson LL, Mather JF, Fowler MS, Ahlberg AW, et al. Risk stratification with electrocardiographic-gated dobutamine stress technetium-99 $\mathrm{m}$ sestamibi single-photon emission tomographic imaging. Value of heart rate response and assessment of left ventricular function. J Am Coll Cardiol 2006;47:781-8.

22. Iskandrian AS, Heo J, Kong B, Lyons E. Effect of exercise level on the ability of thallium-201 tomographic imaging in detecting coronary artery disease: Analysis of 461 patients. J Am Coll Cardiol 1989;14:1477-86.

23. Heller GV, Ahmed I, Tilkemeier PL, Barbour MM, Garber CE. Influence of exercise intensity on the presence, distribution, and size of thallium-201 defects. Am Heart J 1992;123:909-16.

24. Rothembacher D, Hoffmeister A, Brenner H, Koenig W. Physical activity, coronary heart disease, and inflammatory response. Arch Intern Med 2003;163:1200-5.

25. Ulrich L, Wassmann S, Czech T. Physical inactivity increases oxidative stress, endothelial dysfunction, and atherosclerosis. Arterioscler Thromb Vasc Biol 2005;25:809-14.

26. Knowler WC, Barrett-Conner E, Fowler SE, Hamman RF, Lachin $\mathrm{JM}$, Walker EA, et al. Reduction in the incidence of type 2 diabetes with lifestyle intervention or metformin. $\mathrm{N}$ Engl $\mathrm{J}$ Med 2002;346:393-403.

27. Ross R, Janssen I. Physical activity, total and regional obesity: Dose-response considerations. Med Sci Sports Exerc 2001;33: S521-7.

28. Leon AS, Sanchez OA. Meta-analysis of the effects of aerobic exercise training on blood lipids. Circulation 2001;104:II-414-II-5.

29. Fagard RH. Exercise characteristics and the blood response to dynamic physical training. Med Sci Sports Exerc 2001;33: S484-92.

30. Sharir T, Rabinowitz B, Livschitz S, Moelem I, Baron J, Kaplinsky E, et al. Underestimation of extent and severity of coronary artery disease by dipyridamole stress thallium-201 single-photon emission computed tomographic myocardial perfusion imaging in patients taking antianginal drugs. J Am Coll Cardiol 1998;31: $1540-6$.

31. Taillefer R, Ahlberg AW, Masood Y, White I, Lamargese J, Mather $\mathrm{C}$, et al. Acute beta-blockade reduces the extent and severity of myocardial perfusion defects with dipyridamole Tc-99m sestamibi SPECT imaging. J Am Coll Cardiol 2003;42: 1475-83.

32. Berman DS, Kang X, Hayes SW, Hayeas SW, Friedman JD, Cohen I, et al. Adenosine myocardial perfusion single-photon emission computed tomography in women compared with men. Impact of diabetes mellitus on incremental prognostic value and effect on patient management. J Am Coll Cardiol 2003;41: 1125-33.

33. Abidov A, Hachamovitch R, Rozanski A, Hayes SW, Santos MM, Sciammerella MG, et al. Prognostic implications of atrial fibrillation in patients undergoing myocardial perfusion single-photon emission computed tomography. J Am Coll Cardiol 2004;44: 1062-70. 